\title{
Predictive Composition of Pictogram Messages for Users with Autism
}

\author{
Raquel Hervás · Susana Bautista • Gonzalo Méndez • Paloma Galván • \\ Pablo Gervás
}

Received: date / Accepted: date

\begin{abstract}
Communication is a basic need for every person. However, there are many people who present disabilities that prevent communication through natural language. Augmentative and Alternative Communication (AAC) systems, including those based on pictograms, attempt to facilitate the communication for people with this kind of difficulties. In this paper we present PictoEditor, an Augmentative and Alternative Communication application for the composition of pictogram messages for users with autism that incorporates prediction functionalities. Although such functionalities have been widely studied in text-based Augmentative and Alternative Communication tools, they have not been applied to pictogram based ones. The results show that prediction based on frequency of use of specific pictograms improves the immediate availability of the desired pictograms, but the improvement with pre-
\end{abstract}

The work presented in this paper has been funded by the projects IDiLyCo: Digital Inclusion, Language and Communication, Grant. No. TIN2015-66655-R (MINECO/FEDER) and InVITAR-IA: Infraestructuras para la Visibilización, Integración y Transferencia de Aplicaciones y Resultados de Inteligencia Artificial, UCM Grant. No. FEI-EU-17-23.

R. Hervás, P. Galván

Facultad de Informtica, Universidad Complutense de Madrid, Spain

Tel.: +34 913947568

E-mail: \{rhervasb,palomagalvan\}@ucm.es

S. Bautista

Escuela Politcnica Superior, Universidad Francisco de Vitoria, Spain

E-mail: susana.bautista@ufv.es

G. Méndez, P. Gervás

Instituto de Tecnologa del Conocimiento, Universidad Complutense de Madrid, Spain

Tel.: +3491394 7560/7639

E-mail: \{gmendez,pgervas\}@ucm.es diction based on sequencing of pseudo-syntactic types of pictogram is not as clear.

Keywords Augmentative and Alternative Communication (AAC) · Autism · Ergonomics · Interaction Design · Personalization · Pictograms · Prediction

\section{Introduction}

We currently live in a society that could well be defined as a society of information and communication. It is becoming more and more indispensable to have fast communications, as proven by the proliferation of applications for instant messaging in mobile telephones, electronic mail systems, and telecommunication networks. Fast and easy communication is becoming a daily need in enterprise settings, but also in the daily life of ordinary people. Communication becomes a paramount need for everyone - contact with family, communication with friends, event planning. ..-, but there are several populations, people with autism spectrum disorders among others, whose circumstances make it difficult for them to enjoy this kind of communication with the world at large. For these people, and for all those who need to interact with them - such as family, doctors or teachers -, communication is a challenge.

Augmentative and Alternative Communication systems (AAC) arose to meet this need. In particular, the use of pictograms, schematic symbols that represent ideas or concepts, is quite extended in this kind of systems. These pictograms are images representing concepts of all kinds regardless of language, and pictogram users adopt them as a substitute of natural language so they can communicate in an easy and efficient way. There are several sets of pictograms ranging from colour 
or black-and-white illustrations to photographs of specific objects, and a vast number of AAC applications that use them. The high number of pictograms available in these sets constitutes an advantage from the point of view of their expressive power, but is in some ways a disadvantage in terms of their ease of use. To harness the expressive power of extended pictograms sets in a useful way, AAC systems have to find practical ways of organizing the pictograms so the users can easily find them when needed.

In this paper we present the design and evaluation of prediction mechanisms in an AAC system aimed to the composition of messages using pictograms. Text prediction is a widely implemented aid in AAC systems, but pictogram prediction has barely been studied. However, we consider prediction to be more than a convenience in AAC systems, because in many cases the user may not have the intellectual ability to handle the application fluently and find the pictogram sought as would a person who does not present any disability. In addition, whereas most of the applications are fixed to general profiles that try to cover all possibilities for a specific group of people or a particular impairment, our system adapts to each user because it learns the expected usage of pictograms while it is being operated.

The paper is structured as follows. First, in section 2, we introduce the field of Augmentative and Alternative Communication and how prediction has been studied in some of AAC systems. Then, a prediction-based tool for the composition of messages using pictograms is presented in the section 3 . The specific functionalities implemented in the tool for facilitating message composition are explanied in section 4 , and their evaluation is then thoroughly presented and discussed in section 5. Finally, conclusions drawn and intended future work are described in section 6 .

\section{Related Work}

This section presents some related work on Augmentative and Alternative Communication (AAC), with a special focus on applications using the same set of pictograms as our system. In addition, we discuss the importance of prediction in AAC systems, and how this kind of mechanism has barely been implemented in pictogram-based applications.

\subsection{Augmentative and Alternative Communication based on Pictograms}

Augmentative and Alternative Communication (AAC) systems are oriented to users who cannot use natural language in any of its forms due to temporary or permanent impairments, or for whom the use of natural language implies a significant effort. In all cases, this kind of communication tries to provide an alternative to natural language for communication and therefore allows these users to communicate satisfactorily in their daily life.

Many AAC systems rely on visual communication for different target users in different fields. For example, sign language for deaf people is probably one of the best known examples of Augmentative and Alternative Communication. In the last years, there have been some works aiming to translate text to sign language for users whose natural communication channel is vision (San-Segundo et al. 2012; Verdú et al. 2017) and to automatically recognize these signs (Ameen and Vadera 2017; Tao et al. 2018; Khari et al. 2019; Sevilla and Lahoz-Bengoechea 2019).

When considering cognitive impairments, pictograms are one of the most used resources. A pictogram is a schematic symbol that represents an idea or concept in a manner that allows communication across language barriers. Therefore, for people who have this kind of language difficulties, a pictogram is not only a way to express ideas and feelings, but also a tool to interpret, understand and ultimately transform their ideas into pictures in a schematic and efficient way. Thus, pictograms may represent many types of concept: objects, animals, persons, feelings, actions, or even grammatical elements.

Although there is a wide variety of pictogram sets, such as such as Blissymbolics ${ }^{1}, \mathrm{PCS}^{2}$, Pictogram ${ }^{3}$, Widgit $^{4}$, Beta ${ }^{5}$, and Sclera ${ }^{6}$, the most widely used in Spain is the one created by ARASAAC ${ }^{7}$, the Aragonese Portal of Augmentative and Alternative Communication. This is the biggest association working on Augmentative and Alternative Communication in Spain, with the aim of offering resources and materials to facilitate the communication of those with any difficulties in this area. Through their web portal it is possible to find several support products for communication, including technological tools such as programs for computers and tablets which allow alternative ways of access for users with difficulties using language.

Many of these resources are based on pictograms, including a quite complete pictogram database in Span-

\footnotetext{
1 http://blissymbolics.org/

2 https://goboardmaker.com/

3 http://www.pictogram.se/

4 https://widgit.com/

5 https://www.betasymbols.com/

6 http://www.sclera.be/

7 http://www.arasaac.org/
} 


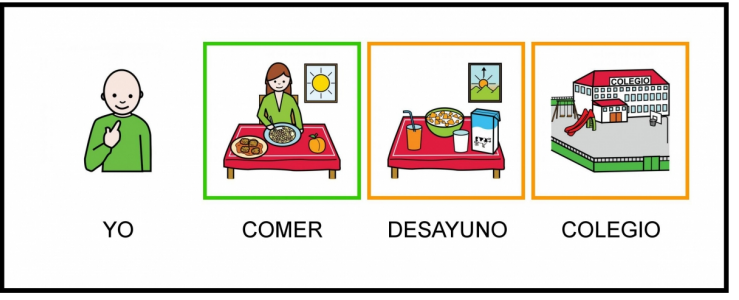

Fig. 1 Example of a sentence using ARASAAC pictograms: yo como el desayuno en el colegio (I eat breakfast at school)

ish that is freely available under the Creative Commons license. In Fig. 1 we can see an example of a sentence expressed using ARASAAC pictograms.

There are several AAC applications based on the pictograms created by ARASAAC for Spanish with the purpose of improving and personalizing the communication and interaction of users who present communication problems.

E-Mintza ${ }^{8}$ means "electronic speech" in Basque, and it consists of a communication board that uses sounds and ARASAAC pictograms. The application classifies the pictograms into semantic classes. When the user selects a pictogram, the corresponding sound is reproduced and the pictogram is added to the message in the blackboard defined in the interface in order to form a sentence. The system is specially designed for users with autism or problems in oral or written communication.

Azahar $^{9}$ is a set of free and adaptive applications to help people with autism or any other cognitive difficulty to improve their communication. The applications allow them to plan their tasks and enjoy their activities. These applications can be used in tablets, computers or smartphones, helping to improve the quality of life and independence of people with autism or intellectual disabilities.

$\mathrm{TICO}^{10}$ is an application for the design and execution of interactive communication boards. This tool has two elements: the editor and the interpreter. Using the editor a board can be created using pictograms, sounds or links to others boards created before. The interpreter allows to execute boards created by the editor in previous sessions.

AraBoard (Baldassarri et al. 2014b) is a set of tools focused on creating pictogram boards using a computer, smartphone or tablet. The aim of this tool is to support the planning of different routines, creating and explaining tasks in an easy way. In addition, personalized pictograms can be used in the boards using images and

\footnotetext{
8 http://www.policlinicagipuzkoa.com/e-mintza/

9 http://www.proyectoazahar.org/

10 http://arasuite.proyectotico.es/index.php
}

photographs. One of these tools is AraWord (Baldassarri et al. 2014a), an application to edit communication boards based on pictograms. AraWord works as a word processor allowing to write at the same time pictograms and text, with the goals of easing the creation of new documents and adapting texts for people with communication difficulties.

AraTraductor (Bautista et al. 2017) is a text-topictogram translator that relies on Natural Language Processing techniques in order to improve the obtained translations. The plain text input is parsed using a syntactic analyzer, and the information obtained from this analysis is then used to create a translation as close as possible to pictogram texts manually created by experts. This tool is implemented in Android so it can be easily accessed through tablets and smartphones, and it also relies on ARASAAC pictograms.

PICTAR (Martín et al. 2018) is another tool on AAC for people with autism, which is also based on ARASAAC pictograms. PICTAR is a web application based on a service of translation from text to pictograms in order to help teachers and tutors in the creation of new pictogram materials from texts like daily routines, stories or news.

A common problem in all these tools is the huge number of pictograms available. Although having a considerable pictogram database allows the communication of a wider variety of ideas, users face a substantial navigation effort in traditional pictogram-based AAC systems due to the number of pictograms to be managed. Most of these systems are organized using some kind of classification of pictograms depending on their topic or meaning, or even the individual preferences of the user or an appropriate configuration for performing a specific task (visiting the doctor or attending school, for example). In addition to this kind of classification, in this paper we will also explore the possibilities of adding prediction mechanisms when using such a system to create pictogram messages.

\subsection{Prediction Techniques in Augmentative and Alternative Communication Systems}

Text prediction methods are the most extended techniques to improve the rate of communication for people who use Augmentative and Alternative Communication systems due to any kind of motor or speech impairments (Garay-Vitoria and Abascal 2006). These methods range from word prediction (Koester and Levine 1996; Venkatagiri 1993; Magnuson and Hunnicutt 2002; Pouplin et al. 2014) to sentence prediction (Arnott et al. 1992; Garay-Vitoria et al. 1995; Mitchell and Sproat 2012; Garcia et al. 2014), through the use of n-grams 
(Wiegand and Patel 2012), topic modeling (Trnka et al. 2006) or context-awareness (Higginbotham et al. 2009; Garcia et al. 2015) as base of the prediction algorithms.

Although several works have demonstrated that different word prediction techniques improve communication rates significantly in AAC (Venkatagiri 1993; Anson et al. 2006; Trnka et al. 2007), only a few instances can be found in the literature where some kind of prediction method has been applied to pictograms.

Gea et al. (2006) describe a location-aware Augmentative and Alternative Communication tool for children with autism. Symbols are presented in the interface according to a set of rules that take into account user location and semantic networks created by teachers and rehabilitation professionals. Although the authors do not report any evaluation of the system, they mention that they are working in scenarios such as the child's house, school and other specific locations (e.g. restaurants).

Kim et al. (2009) propose a location-aware system based on pictograms that consider the context in which the communication is taking place. This context is defined by the user location (e.g., a restaurant) and a communication goal (e.g., ordering), and the system relies on a server that contains a knowledge model describing relations between pictograms and locations, and pictograms and goals. The frequency of use of each pictogram is also stored in the server.

García et al. (2015) present the design of a pictogram communicator for people with speech impairments that is able to spontaneously adapt to each user profile and be aware of the situation: location, time of the day and interlocutor. Therefore, the vocabulary to be used relates to a particular situation with the possibility to be broadened by the user if needed. However, although their design is very promising and the future capabilities of the communicator quite interesting, the authors recognize that it is only a design and they have not developed the intended system yet.

In (Garcia et al. 2016) the authors evaluated the performance of Eugénio, an AAC system for Portuguese, by measuring the effects of adding location knowledge to its pictogram prediction system. The location-aware pictogram prediction method was trained using a corpus composed by able-bodied persons in three different locations: classroom, cafeteria and home. Although their results were promising, they recognize that their solution is based on a corpus that was created by people with no disabilities, which could bias the results.

Pahisa Sol (2017) presents a system that transforms the telegraphic language that comes from the use of pictogram-based AAC into natural language in Catalan and Spanish. In order to test this idea, a user interface with pictogram prediction was implemented. This prediction is based on n-grams, syntactic-semantic features and context information.

In summary, while there is extensive work in text prediction for AAC systems, to the best of our knowledge there are only a few pictogram prediction initiatives. However, they mostly tackle prediction from the perspective of anticipating specific contexts, rather than using a pure text-prediction approach as the one proposed in this work.

\section{PictoEditor: an AAC Application for the Composition of Pictogram Messages}

PictoEditor is an AAC system, implemented as a tablet app, intended to help users who need to compose a sentence or message to communicate through pictograms with persons with Autism Spectrum Disorder. It has been designed for Spanish users, so it relies on the ARASAAC set of pictograms and all pictograms in the application are accompanied by their corresponding words and sounds in Spanish.

All ARASAAC pictograms are accessible through different means, so the user can find them as easily as possible. In addition, the finished messages or sentences can be shared with other applications (chat, mail, ...) through the sharing capabilities of the Android operating system. The tablet app does not contain the whole ARASAAC database, but only the pictograms loaded by the user, and it relies on a server where the complete database is stored.

As in the case of other cognitive disabilities, such as Down syndrome, for whom there are no specific guidelines that can be used to design applications (AlonsoVirgos et al. 2018), it is difficult to find such guidelines for users with autism. Therefore, PictoEditor has been designed following a User Centered Design (UCD) involving experts, which is a method recommended by several authors, not only because they can enhance the communication with end users, but because they provide a wider context of the needs and problems faced by a collective of people with impairments that can be important in the development process (Allen et al. 2008; Keay-Bright 2007; Kientz et al. 2007; van Rijn and Stappers 2008).

UCD has been favoured over Participatory Design (PD) as, even though some authors suggest it may be beneficial (Pantsar-Syväniemi et al. 2015), other authors have reported difficulties when using PD, such as end users being unable or even unwilling to collaborate (Holone and Herstad 2013; Allsop 2010), specially when end users are children, for whom the involvement 


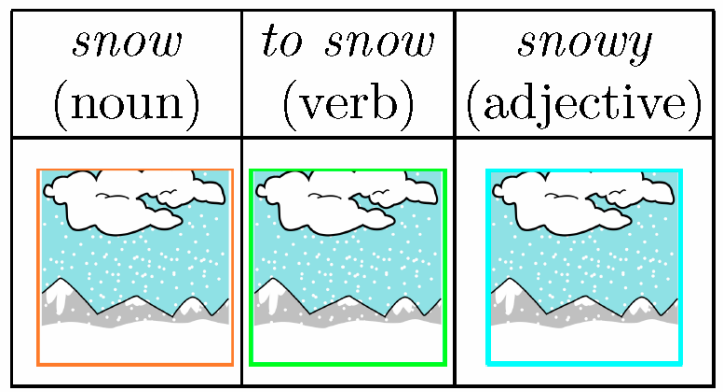

Fig. 2 Same pictogram with different meanings depending the POS role in which they are employed, distinguished by the coloured frame

required by $\mathrm{PD}$ may be too big (Frauenberger et al. 2011).

\subsection{ARASAAC Pictogram Database}

In order to prepare a tool that helps users to create messages in pictograms as a mean of communication with persons with Autism Spectrum Disorders, we need to use a set of pictograms that is widely known for them. We have decided to use the pictograms from ARASAAC that are widely used in Spain and contain approximately a total of 8500 pictograms corresponding to a vocabulary of more than 15000 words. Each pictogram is associated to a number of words in different languages: Spanish, English, German, Italian, French, Basque and Catalan. These words correspond to the things or ideas that are represented by the pictogram. For example, the same pictogram is used for nieve (snow), nevar (to snow), nevada (snowfall), nevado (snowy) and tormenta de nieve (snow storm). In our application we work only with Spanish words, but the database would let us work on different languages by introducing small adjustments in the tool.

In addition to pictograms and words, the database stores a colour for each word corresponding to a pictogram. This colour represents a pseudo-syntactic category for the pictogram following this equivalence:

- Orange - Common nouns

- Yellow - Proper nouns

- Green - Actions/Verbs

- Blue - Adjectives

- Pink - Social

- White - Miscellany

These categories described in terms of colours have a rough correspondence with Part-of-speech (POS) tags as considered in Natural Language Processing applications. This POS colour of a pictogram is represented by a coloured frame surrounding the image. Fig. 2 shows the framed pictograms corresponding to the snow pictogram depending on the meaning it is representing.

The process followed to transform these data into the material required by our project was somewhat laborious. As extensive and structured as the ARASAAC database is, it does not organize the pictograms into any kind of topics or semantic classes. However, this was essential for our system, as this kind of communicators usually arrange pictograms in this way so it is easier to find the desired pictograms at any moment. Therefore, we manually assigned to each pictogram a thematic class, in such a way that the set of pictograms became distributed into balanced groups. The thematic classes range from people or nature to sports or technology.

\subsection{System Description}

The application works on any Android device, but it is specially intended for tablets and to be used in landscape mode. The first time the application is started, a selection of the most common pictograms is downloaded into the device. As we will see later, the user can afterwards download any ARASAAC pictogram that he might need. The main working screen of the application can be seen in Fig. 3.

The top part of the screen corresponds to the blackboard where the user will compose the sentence. It has the restriction of a maximum of 12 images, which we have considered sufficient for a normal phrase, and has a slider bar for cases in which they cannot be visualized at the same time. As the user presses any pictogram, it is added to the blackboard along with its corresponding word below it, and the system reads aloud this word in Spanish. The user can also delete the last pictogram by pressing the button to the right of the blackboard, which is the pictogram for "erase".

The central and largest area corresponds to the pictogram selection box. This area is a table with nine tabs, one for each thematic class. The area under the tabs shows the pictograms contained in the device or the server corresponding to the class/tab selected (with a maximum capacity of 30 pictograms). Its behaviour varies depending on whether the application is in write mode or load mode.

When the application is in write mode, the pictogram selection box contains the pictograms the user has loaded and used in the past, along with the pictograms stored from the initial load. Once a pictogram is pressed, it will be added to the message being created. The user can also erase a pictogram by pressing on it for a time. A confirmation dialog will be displayed and - if the user 


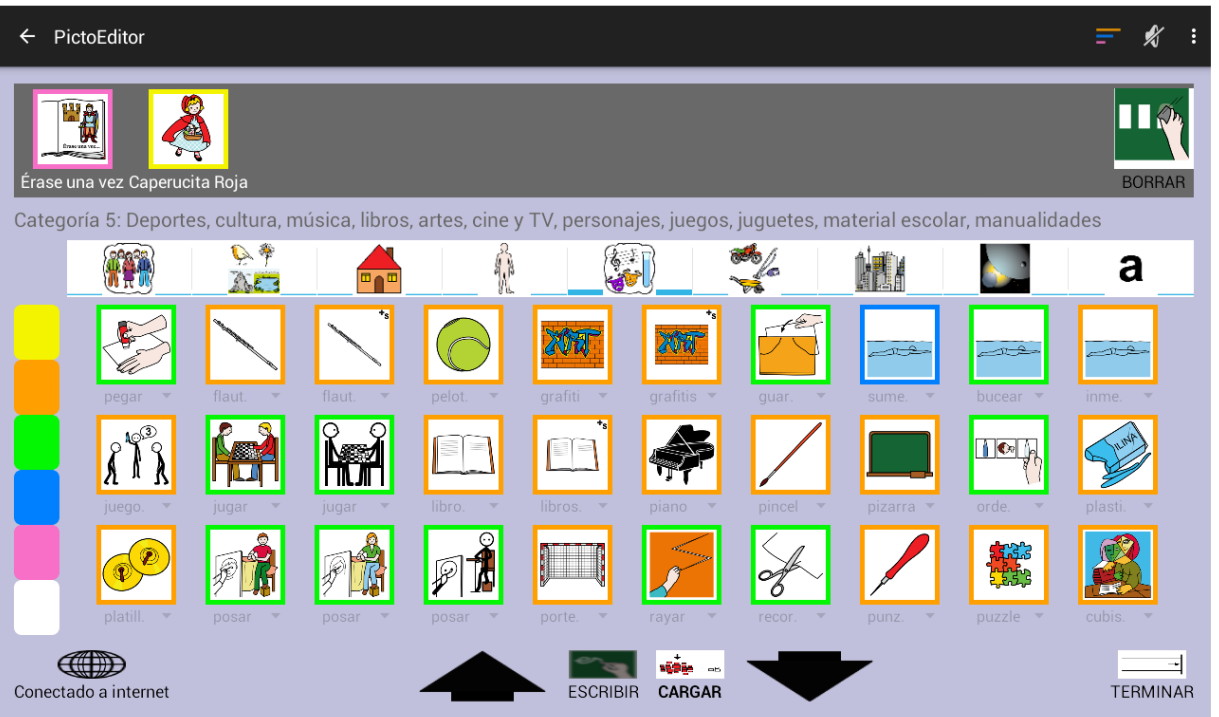

Fig. 3 Working screen of the PictoEditor application

accepts - the corresponding pictogram will be removed from the storage of the device. If the user wants to recover the pictogram at a later stage, he would have to use the pictogram load mode.

The purpose of load mode is to allow the user to load pictograms from the server, which are displayed in the pictogram selection box. When one of these pictograms is pressed, the file for it is transferred to the local storage in the device. This makes the corresponding pictogram available to the user during write mode. When entering load mode, thirty pictograms are loaded for each class whenever there is internet connection, which is necessary for this mode to work.

In addition, to the left of the pictogram selection box we find a section with the POS colours of ARASAAC (explained in the previous subsection). These correspond to the colour shortcuts we will describe later. Each colour button shortcut interacts with the pictogram selection box, ensuring that the pictograms matching the chosen colour are shifted to the first positions of the box.

Finally, at the bottom of the main screen there are a series of buttons and icons. The first is an icon that informs the user if the application has internet connection or not. Besides we can find four navigation buttons: two black arrows - that change the contents of the pictogram selection box following a page system -, the button to switch to write mode and the button to switch to load mode. The button corresponding to the selected mode is not shaded, and its text is highlighted in bold and italic. The Finish button redirects the user to the final screen, where the composed message is displayed and different actions are offered.
In the final screen a single image is constructed by collating the pictograms that had been inserted in the blackboard of the edition screen. As we can see in Fig. 4 , each pictogram appears with its corresponding Spanish word, so the phrase is easily readable. This screen also offers two actions on the displayed image: Save and Share. If the sentence is saved, it is stored in the external memory of the device, so it can be opened by any application working with images. If the message is shared, the application displays a window which shows all the applications available on the device through which an image could be shared. This way the user could establish a communication through an instant messenger client available on his device, or attach the image to an e-mail, for example.

\section{Specific Functionalities for Facilitating Message Composition}

Three specific functionalities have been added to the system to facilitate the composition of pictogram messages. Of these, two rely on the ability to predict, based on the record of prior interactions with the system by the current user, which pictograms are more likely to be required as continuation of an ongoing message. These, when activated, operate automatically, rearranging the way in which the available pictograms are displayed for the user to employ. The other functionality can be invoked explicitly by the user and it allows specific recall of pictograms of a given POS colour category. This allows the user to bring to the fore pictograms other than those predicted as possible continuations by the system. 


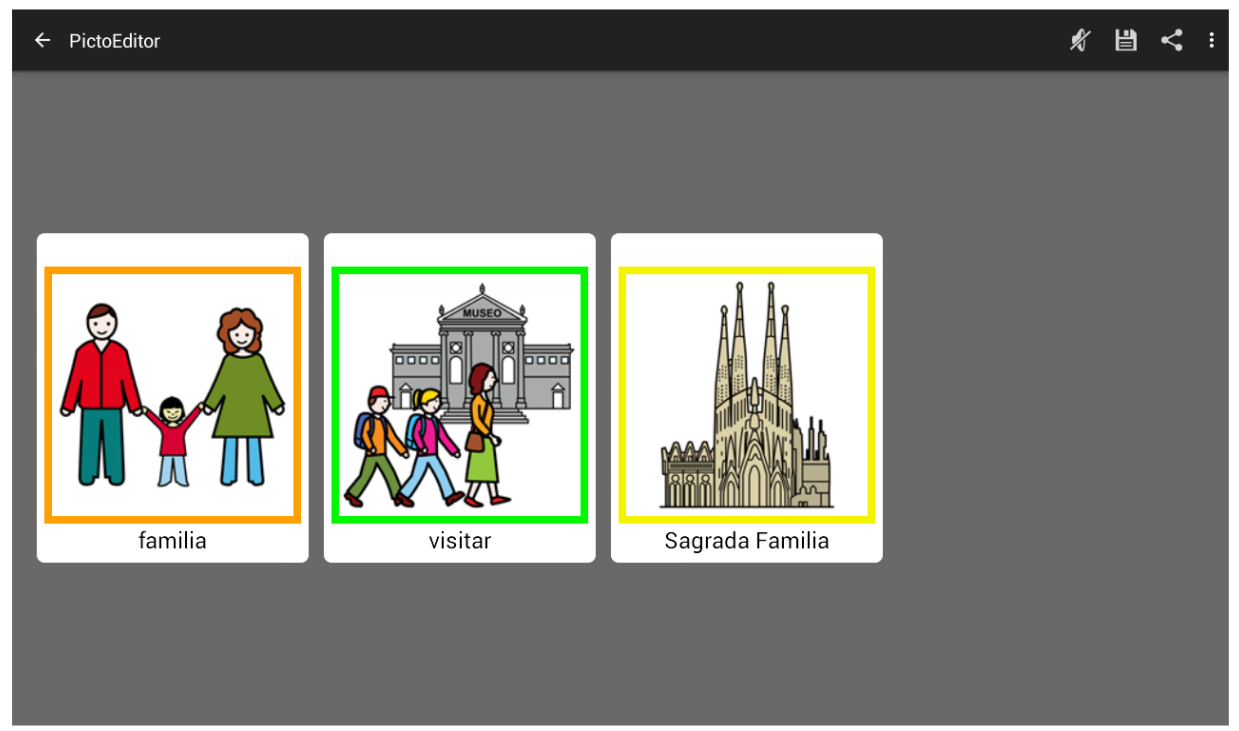

Fig. 4 Final message composed using the tool

\subsection{Pictogram prediction based on usage statistics}

We have included in the application a predictive algorithm to facilitate the composition of sentences by the user. This algorithm is based on the recorded history of pictogram sequences constructed by the user in the past. The theory underlying this approach is that of n-gram models, where an n-gram is a sequence of $n$ consecutive words or tokens that appear together in a text or a sequence of elements. N-gram models are used in our application to register statistics of use at two different levels: the number of instances of use recorded for each pictogram, and the POS colours of ARASAAC which correspond to syntactic categories associated with the corresponding pictogram.

\subsubsection{Prediction based on Pictogram Frequency of Use}

Each time the user selects a pictogram in write mode, the number of instances of use recorded for that pictogram is internally increased. This value is stored in an internal data structure of the application. As the tool is handled by a given user, the frequency of use of each pictogram is registered in this way. This provides data for a unigram model of pictogram sequence, or $\mathrm{n}$-gram model of the sequences of pictograms of order 1.

Fig. 5 shows a sentence under construction. Pictograms with a greater number of instances of use appear earlier in the arrangement within their POS colour, so that the blue pictogram that has just been included earlier in the messages appears as the first of the blue pictograms, and the most frequently used of orange pictograms appear as the firsts of their set.

\subsubsection{Prediction based on Part-of-speech Colour Sequencing}

The PictoEditor application also builds a bigram model of sequences of POS colours of pictograms (explained in Section ARASAAC Pictogram Database) as composed by the user. This is done by registering the frequency in which subsequences of two colours appear consecutively in a message, which corresponds to an n-gram model of order 2 built at the level of pictogram POS colours (which represent syntactic categories). In this way, the system learns from the user, establishing relationships between the POS colour categories to facilitate sentence composition. The algorithm comes into action each time the user clicks on a pictogram in write mode. Each time this happens, the pictograms of the pictogram selection box are sorted according to their colour and the colour sequence data collected. In addition, within the order by colour they are ordered by number of instances of use so that the composition of routine phrases is facilitated.

Fig. 5 can also be used to explain the mechanism for POS colour based prediction. The last pictogram added to the message has been a noun (orange) so the algorithm reorders the pictogram selection box to display first the adjectives (blue), then nouns (orange) and finally the verbs (green). This is based on the relative ngram probabilities for orange-blue, orange-orange, and orange-green subsequences. This example also illustrates how the two different mechanisms for pictogram prediction interact when the tools is used.

One must keep in mind that these data depend on the user and his habits, and that writing with pictograms often does not follow the logic to which we 


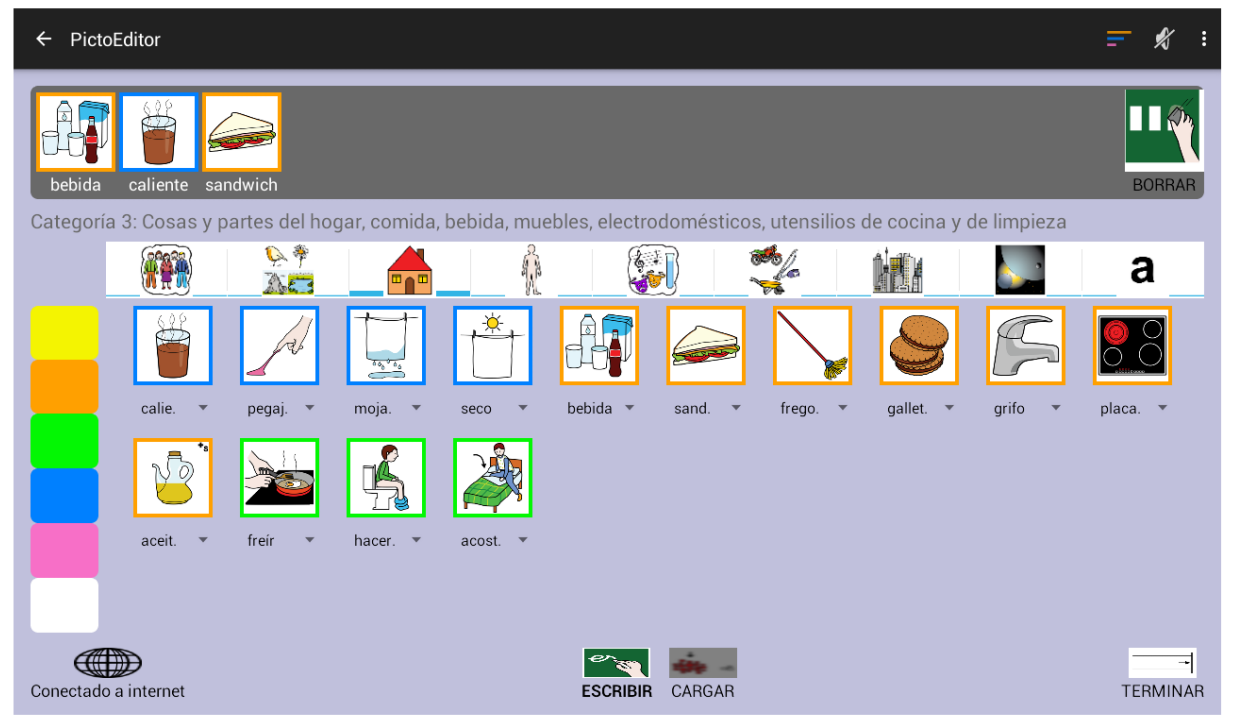

Fig. 5 Example of frequency and POS colour based prediction

are accustomed. For example, in Spanish we would say "The garden is beautiful" while the corresponding sentence with pictograms transcribed into Spanish could be "The garden is pretty", "Garden is pretty" or "Pretty garden" simply. As seen, in the first two cases, a noun is followed by a verb, but in the latter the noun "garden" is followed by an adjective, omitting the verb.

\subsection{POS colour shortcuts}

Every user of mobile phones knows that predictive input systems and orthographic correctors can sometimes be persistent in providing incorrect or unwanted options. This is a risk that may also affect the predictive solutions we are considering. To help the user override the predictive functionalities in certain cases, the application includes the functionality for POS colour shortcuts. Colour shortcuts are invoked from the vertical bar with the POS colours of ARASAAC that appears to the left of the pictogram selection box (explained in Section System Description). When a given colour is pressed on this bar, the pictograms matching the chosen colour are shifted to the first positions of the pictogram selection box. This allows the user to override the prediction based on pictogram colour categories when it does not match his intentions.

This has proven to be very useful, possibly due to the fact that pictogram communication does not follow rules of syntactic sequencing of pictogram types as rigidly as natural language.

\section{Evaluation}

We have conducted an evaluation of the described tool in order to assess the effectiveness of the predictive algorithms previously described, based on the frequency of use of the pictograms and based on their POS colour category, so that we can decide whether to include them or not in the final version of the editor. More specifically, the aim of the evaluation was twofold: first, our purpose was to test the usefulness of different combinations of the predictive algorithms regarding how easy it is, from the user's point of view, to find the next pictogram to be used when composing a message; second, we intended to evaluate how the predictive algorithms help the users to improve their effectiveness and efficiency to complete a task in terms of the time required to carry it out. Additionally, at this stage of the development, we were also interested in assessing the interaction and usability of the editor in order to improve them in the final version of the editor. In the following subsections we describe the conditions under which we have conducted this evaluation, including the description of the participants, the materials and the method, along with the results we have obtained.

\subsection{Participants}

Our objective was for the editor to be used to improve the communication between children with autism and their family and teachers. As stated by Hervás et al. (2019), it is usually difficult to work with users with cognitive disabilities. The problem is not only that these users may face extra difficulties to express their needs 
or desires, but also that they may not be available to be involved during such complex and usually long processes. Therefore, it is important to employ usercentered methodologies that rely less on the availability of these users and can take advantage of other stakeholders, such as experts, caregivers or tutors, who can act as their proxies. One of the stakeholders who has helped us define the requirements under which the editor should work is a teacher in a school for children with autism spectrum disorders. The children this teacher works with have different ages, but they all have a low cognitive level and severe communication difficulties. For this reason the evaluation was not carried out directly with the children, but with the teacher since, in her own words "they find the tablets amusing, but they are still not able to work with them". Therefore, the target subject of this evaluation has been a teacher of children with autism spectrum disorder, female, around 30 years old, familiar with the use of tablet devices and skilled in the use of other applications based on pictograms.

\subsection{Materials}

Every day, when the children arrive to school, they gather in an assembly in class where the teacher explains to them what they are going to do that day. The structure of what they do in the assembly is always the same, so that they can feel the situation is under control and they do not get nervous. First, they write the current date and what the weather is like that morning. After that, they write in strict order the tasks they are going to carry out that day (e.g. say hello, wash their hands, have lunch, and so on) and, finally, they remember the rules they have to follow in class and at school (e.g. no shouting and no fighting).

The aim of the evaluation was to assess if the implemented pictogram prediction mechanisms work properly and could be useful in real tasks involving pictogram messages, in this case, to facilitate the task of the teacher who has to prepare lots of pictogram materials for the class. With this objective in mind, we have adapted the editor for the assembly with the kids: the nine initial thematic classes have been substituted by only four corresponding to the assembly, and the pictograms in the pictogram selection box are bigger so they can be more easily distinguished by the children. This version of the editor has been initially loaded with a set of pictograms that can be useful for the assembly, together with a set of random pictograms that add noise so that the effectiveness of the learning and predictive mechanisms can be tested. In addition, the editor is completely untrained, so that we can test how well the algorithms work from scratch.

\subsection{Methods and Tasks}

In order to test the editor, the task to be carried out has been to reproduce the assembly that takes place in the class each day for over five weeks. We have used different configurations for each week, so that we were able to test which combination of features worked best. Although the evaluation has been done with only one expert, it has been performed in a real-life scenario, and the number of pictograms employed is quite high (around 300) and therefore significant from the point of view of pictogram prediction.

\subsubsection{Evaluation setup}

The features that could be turned on and off were the ones explained in Section Specific Functionalities: the use of the prediction based on the frequency of use of the pictograms, the use of the prediction based on the POS colour category of the next pictogram to be used and the colour buttons that can be used as shortcuts to show the pictograms with that colour. Our initial hypothesis, in order not to make the evaluation unnecessarily long, was that the frequency based prediction improves the results obtained if no prediction is used at all. As commented in the Related Work section, several works have demonstrated that word prediction techniques enhance communication rates in Augmentative and Alternative Communication (Venkatagiri 1993; Anson et al. 2006; Trnka et al. 2007). However, there are no such studies for AAC systems based on pictograms. Therefore, our hypothesis is also based on our previous experience working with people with cognitive impairments, who have problems for finding and choosing the adequate pictogram when they have such an extensive choice (the ARASAAC database contains more than 8,500 pictograms). The special education teacher who evaluated the tool also confirmed this problem. As a result, the frequency based prediction is always on, and the purpose is to test what combination of the other two features provides better results once the algorithms are trained. This gives rise to five sets of tasks, the first one to train the algorithms and the other four to test each combination of activated or deactivated features.

The tasks to be carried out by the teacher, using the different combinations of features, was to write all the messages she would need for the assemblies of five consecutive weeks, i.e. write in order the messages she would need to present the date, weather, day plan and class rules during 25 class days. We have chosen the 
Table 1 Configuration of the tested functionalities for each of the five weeks used in the experiment

\begin{tabular}{lccccc}
\hline & W1 & W2 & W3 & W4 & W5 \\
\hline Prediction based on Pictogram Frequency of Use & ON & ON & ON & ON & ON \\
\hline Prediction based on POS Colour Sequencing & ON & ON & ON & OFF & OFF \\
\hline POS colour shortcuts & OFF & ON & OFF & OFF & ON \\
\hline
\end{tabular}

following configurations of the editor for each of the five weeks (see also Table 1):

- Week 1: since at the beginning the editor is untrained, no reliable prediction can be made until the editor has been able to gather enough data. For this reason, we have enabled both the frequency based prediction and the POS colour based prediction, so that both models can be trained. The POS colour shortcuts have been disabled here so that we can establish a baseline about the number of clicks the user needed to write the messages. In addition, the results for this week can also be used as a baseline for the effectiveness of the prediction algorithms, since we can compare the results of using a trained model against the results of using the initial untrained model.

- Week 2: once the models are partially trained, the messages to be written in this week had all the features on (both predictive algorithms and the POS colour shortcuts), which is supposed to be the most effective configuration to help the user.

- Week 3: in this week we used the same configuration as in the first week, so that we can compare how this configuration works with and without a trained system.

- Week 4: for this week, both the POS colour based prediction and the POS colour shortcuts are disabled, so that we can test how well the frequency based prediction works alone with a trained model.

- Week 5: finally, we tested the system with only the POS colour based prediction disabled, so that we could check whether this feature effectively helps the user to edit the messages or not.

\subsection{Evaluation execution}

The evaluation took place in the school where the teacher works, when the children were not in the class. She was provided with a tablet with the editor and the evaluation was recorded so that all the process could be subsequently analysed. After the evaluation, the teacher was asked to fill in a questionnaire rating different aspects of the editor and providing feedback about its features.
Week 1. As it has been previously described, the task to be carried out this week involved the use of the editor with both the frequency based and POS colour based predictions enabled. Therefore, the pictograms were arranged in no particular order, and there were random useless pictograms in the local database to test the utility of the training.

When writing the date for the first day, the pictograms the teacher needs are scattered through the first four screens, so she has to scroll up and down several times in order to find the ones she is looking for (four pictograms for the week day, month day, month and year). The same happens with the weather. When composing the message for the day plan, where she uses 12 different pictograms, the system shows them according to their colour: orange (nouns), pink (social), yellow (proper nouns) and green (verbs), but as she starts using them the system already arranges them according to their use. As is turns out, most of the pictograms she needs are orange (nouns), so all the orange pictograms remain in the first screen. Finally, she composes the message to write the rules, and she explains that the rules are always the same, so she will not need to write this message for the rest of the evaluation.

After finishing the first day, she proceeds with the messages for the second day, and then for the third. When writing the date for the third day, the month and year are already situated in the first row of the first screen, so she does not have to scroll down to look for them to compose the message. She then completes the messages for the third and fourth days, and when composing the messages for the fifth day it is already obvious that the system is arranging the pictograms according to their use, so most of the pictograms she needs, which are orange (nouns), are already situated in the first screen and she does not need to scroll down to find them. The exception for this are the pictograms to say "hello" and "goodbye", which belong to a different category (pink - social) and the pictogram for "work", which also belongs to a different category (green - verbs).

After finishing the messages for this week, which has taken her 19 minutes, we have been able to see that the system behaviour has been evolving with use, so that after a while the most frequently used pictograms have 
started to appear in the first screen, which has allowed the teacher not to scroll so much as the she had to do during the first two days. However, we have also been able to see that the POS colour based prediction tends to move to the first screen some pictograms of the appropriate colour which, in turn, are never used to compose messages (e.g. "comic book" or "birthday").

Week 2. For the task to be carried out this week, all the mechanisms designed to assist the user have been enabled, so the teacher can count on the frequency and POS colour based prediction, in addition to the shortcut buttons to select the pictograms by colour (in case they are not in the first screen).

The date and weather are created smoothly, with no remarkable differences with the ones of the previous week. When having to create the day plan, she selects the pink colour (social) in order to find the pictogram corresponding to "hello", which is not visible, and then returns to the first screen.

The pictograms for the first day of this week can all be found in the first screen, except for a yellow (proper nouns) one (corresponding to the "eTwinning" activity, an initiative of the European Commission that aims to encourage European schools to collaborate using Information and Communication Technologies (ICT)) which is in the third screen. After doing this, the POS colour based prediction suggests the use of a pink (social) pictogram, situating them in the first row of the first screen, which was learnt during the previous week. During the rest of the days the editor behaves accordingly, so most of the needed pictograms are already in the first screen, except for one on the second day ("library") and another one the fourth day ("work").

The time needed to compose all the messages for this second week has been 9 minutes, which is remarkably shorter than the time used for the previous week.

Week 3. The configuration used this week is the same one used in the first week, although this time the editor is already trained. For all the days of this week, the date and the weather are composed easily. Regarding the day plans, everything works just like the previous week, but the pictograms for "hello", "goodbye" and "eTwinning" are never placed in the first screen, so the teacher always needs to look for them. In addition, in the fourth day she also needs to look for the pictogram for "work", which is green (verbs) and is not placed in the first screen because, in general, an orange (nouns) pictogram is followed by another orange pictogram instead of a green one, so the POS colour based prediction does not help here, and the frequency based prediction prioritizes other pictograms rather than this one.
This week the task is completed in slightly less than 8 minutes, in contrast with the 19 minutes that were required the first week using the same configuration.

Week 4. This time, only the frequency based prediction is active, so no help is provided regarding the POS colour of the pictograms. Therefore, pictograms of all the colours are initially present in the first screen. The date and the weather, again, are created smoothly, with the necessary pictograms appearing in the first screen of the editor. Most of the pictograms needed for the first day are in the first screen, except for the one for "eTwinning". Even "hello" and "goodbye" are in the first screen, since they are promoted by the frequency of use and are not penalized for not having the appropriate colour. The same effect can be appreciated during the second, third and fourth days, when only one pictogram each day have to be found in other screens (namely "library", "handicraft" and "dog therapy", respectively). Regarding the fifth day, all the pictograms are placed in the first screen.

The time taken to complete the task this time is 6 minutes, which suggests that the frequency based prediction may work better when not complemented by the colour based prediction.

Week 5. The configuration used this week adds the POS colour shortcut buttons to the frequency based prediction. Once more, the date and weather messages are created smoothly. As for the day plans, during the first day, all the pictograms are located in the first screen, except for the "eTwinning" one, which is easily found touching the yellow button. In the second day, only the "goodbye" pictogram is not present in the first screen, and is also easily located using the pink shortcut. In the third day it is the "handicraft" pictogram which has to be looked for using the POS colour shortcut. Even so, the pictogram is not placed in the first screen because other pictograms have a higher priority, so the teacher has to scroll down to the second screen in order to locate it. During the fourth day, she needs to use the orange shortcut to locate "dog therapy" and the green one to locate "work". As for the fifth day, all the pictograms she needs can be found in the first screen.

The time needed for the task this week is again 6 minutes, which seems to confirm that the colour based prediction, which has not been used this week, may make the task a little less efficient.

Figs. 6 and 7 show a comparison of the distribution of pictograms in the screen before and after training (weeks 1 and 5, respectively). In both cases the sentence to be composed for Monday is the same (shown in Fig. 8), so it is easy to compare how the prediction 


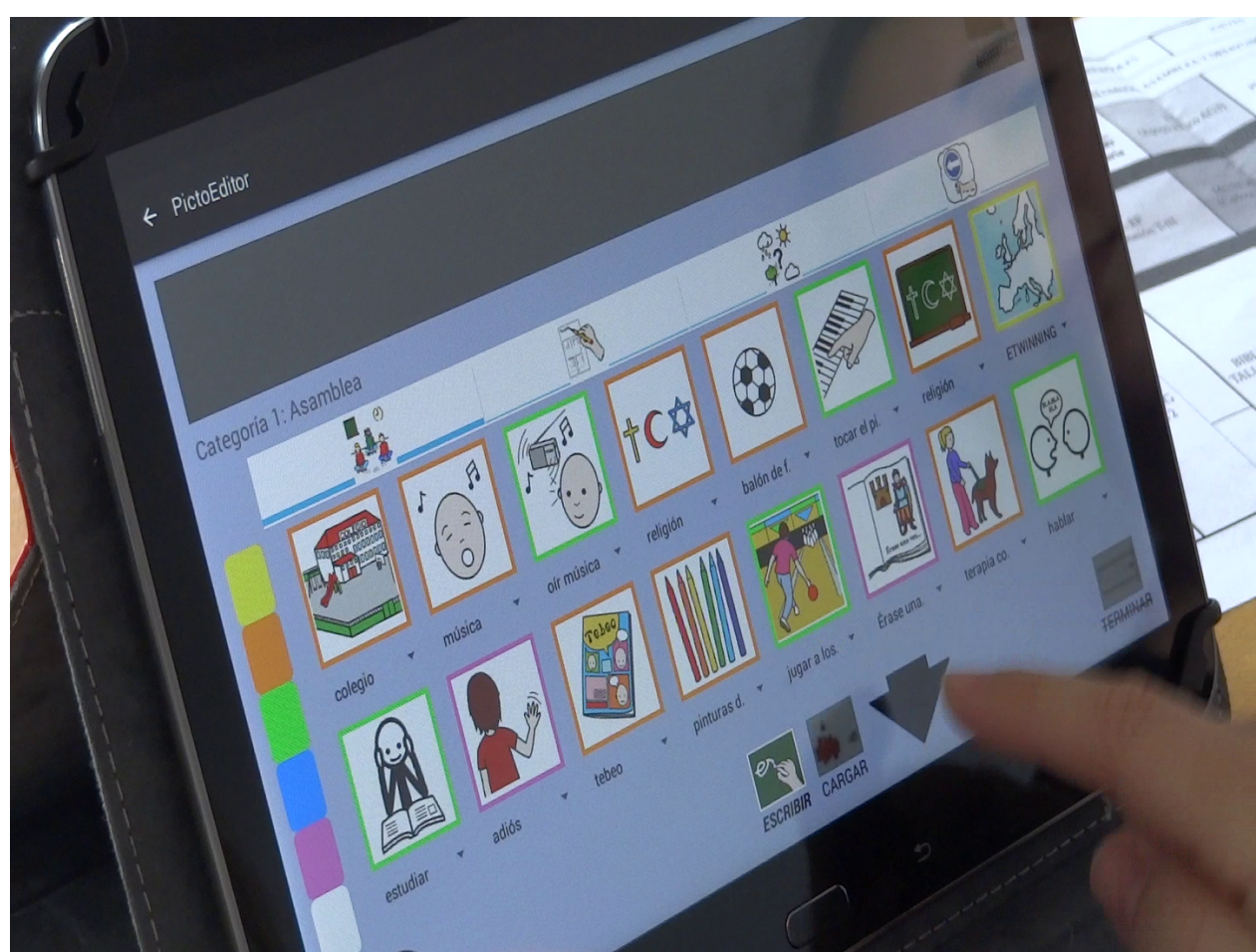

Fig. 6 Distribution of pictograms at the beginning of week 1

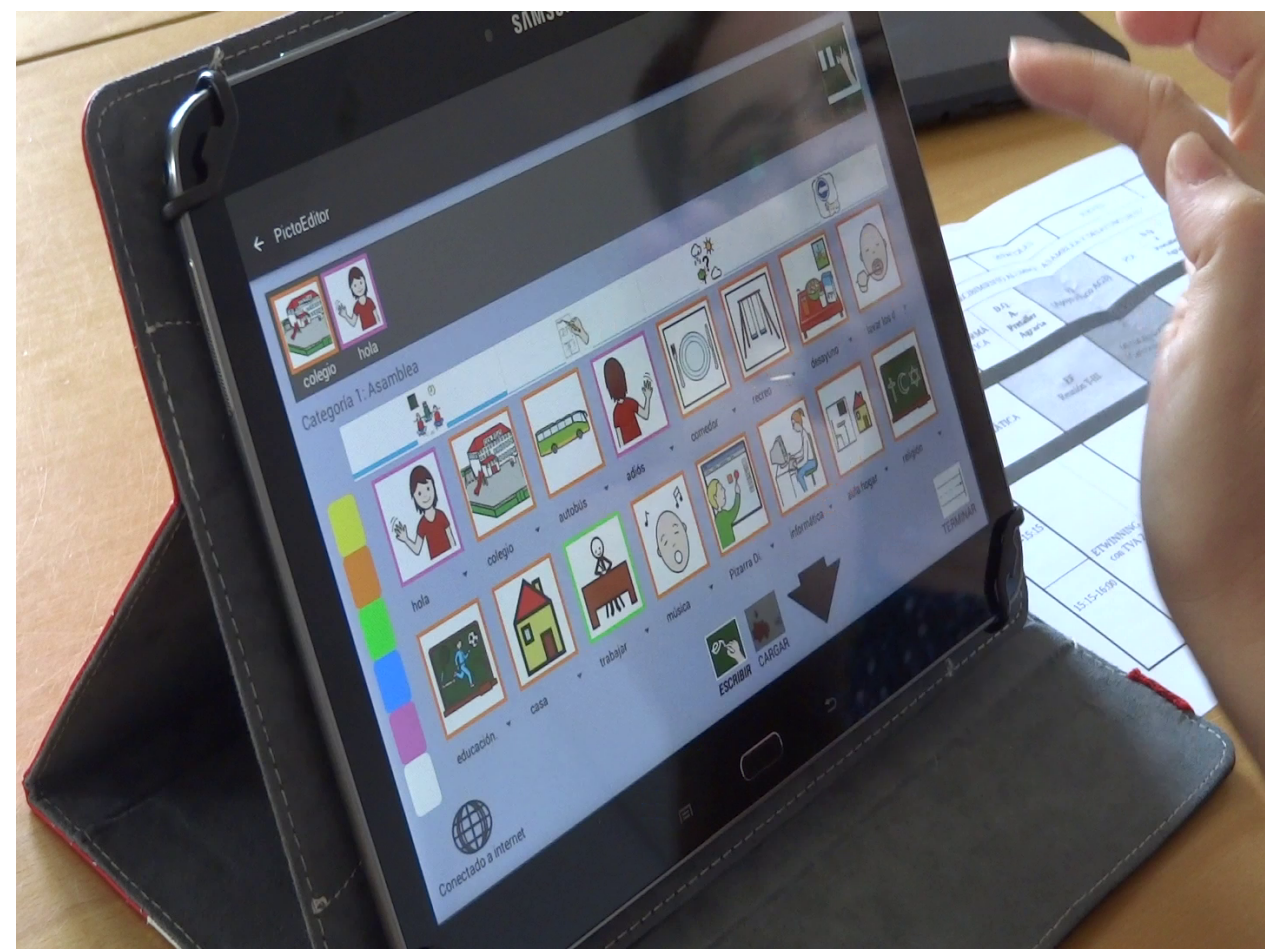

Fig. 7 Distribution of pictograms at the beginning of week 5

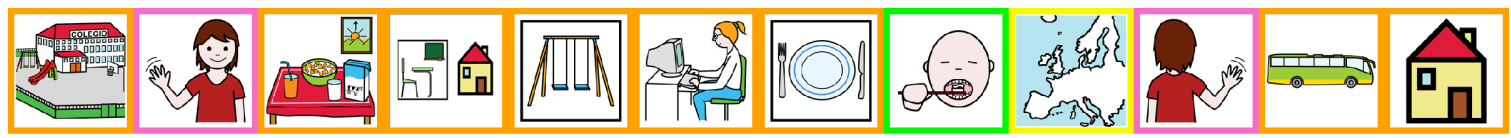

Fig. 8 Example of assembly message for Mondays of weeks 1 and 5. 
is positioning almost all the required pictograms in the first screen in week 5 .

\subsubsection{Questionnaire}

After running the evaluation, the teacher was asked to fill in a questionnaire in order to rate different aspects of the editor.

The first set of questions was intended to evaluate the user interface of the editor. All in all, the user is highly satisfied with the user interface of the application, although she suggests increasing the size of the pictograms can make it easier to find them in the screen.

The second set of questions is aimed at rating the functionality of the editor, where her opinion is that, in general, the predictive algorithms make it really easy to compose the messages, in comparison with other pictogram editors she has used that do not provide this functionality. The teacher also considered that the tool could be useful for users with autism that require pictograms to communicate, although not directly for her class as her children have a very low cognitive level. However, for users with medium and high cognitive levels it would be a great help when working with big amounts of pictograms as is usually the case.

The next set of questions evaluates her satisfaction with the effectiveness and efficiency to complete the tasks of the evaluation using the editor. Again, her impression is that it has been easy to complete the tasks although it is difficult to compare the use of the editor against doing it manually, since the process, in her opinion, is completely different. However, she appreciates the possibility to save and share the created messages, since that way she can prepare the assembly beforehand and it may allow her to carry out other activities with the children during the assembly.

As for the usability of the application, she is satisfied with the way in which the predictive algorithms help her write the messages, and she is willing to use the editor for other tasks than just preparing the assembly, such as general communication with the kids, learning new vocabulary or practicing reading.

Finally, when asked about her satisfaction with the general use of the editor, she scores the maximum punctuation in all questions about the interface, functionalities and ease of use.

\subsection{Results and discussion}

We have divided the results obtained into two different parts: quantitative and qualitative results.

\subsubsection{Quantitative Results}

In Table 2 we can see some numeric data obtained from the evaluation tasks performed during each week.

The first three rows show the configuration of the editor for each one of the weeks as in Table 1, where the different combinations of options we have used are set as $\mathrm{ON} / \mathrm{OFF}$.

The row "Number of pictograms used" refers to the number of pictograms that have been used to compose the class plan during the whole week, taking into account that many of these pictograms are used every day. The number of pictograms used each day ranges from 11 to 13 , giving a total that ranges between 61 and 63 pictograms per week.

Out of these pictograms, the next row of the table shows the number and percentage of them that could be found in the first screen of the editor. It is interesting to see that this number increases significantly when the POS colour based prediction is turned off in weeks 4 and 5 . In contrast, the number of pictograms that can be found in the first row stabilizes around the third week, when the POS colour based prediction is still turned on. The difference in the percentages of pictograms in the first screen corresponds to an average of two pictograms per day, usually the ones needed to say "hello" or "eTwinning". When the POS colour based prediction is used, these two pictograms, which are green (verbs) and yellow (proper nouns), usually come after an orange (nouns) pictogram, but the POS colour based prediction gives a higher priority to an orange pictogram being used after another orange pictogram, since this is what usually happens when composing the messages for the class plan. Therefore, all the orange pictograms are placed before the green and yellow ones, thus causing green and yellow pictograms to be out of the first screen. When only the frequency based prediction is used, the orange pictograms that are scarcely used are given less priority, thus disappearing from the first screen and leaving enough free space for the others to be situated there.

In contrast, the pictograms with different colour than orange (nouns) are used less frequently than the orange ones and so the number of "Pictograms in the first row" remains stable even though the POS colour based prediction is turned off, since the first row is occupied by the pictograms that are used every day and, among them, the ones that have been used more recently. In turn, this is what causes that the number of "Pictograms in the first position" is so low until it goes down to 0 .

When the POS colour based prediction is turned on, the row "Pictogram colour first" shows the number of 
Table 2 Complete results obtained from the evaluation

\begin{tabular}{lccccc}
\hline & W1 & W2 & W3 & W4 & W5 \\
\hline Prediction based on Pictogram Frequency of Use & ON & ON & ON & ON & ON \\
\hline Prediction based on POS Colour Sequencing & ON & ON & ON & OFF & OFF \\
\hline POS colour shortcuts & OFF & ON & OFF & OFF & ON \\
\hline Number of pictograms used & 61 & 62 & 63 & 63 & 63 \\
\hline Pictograms in first screen & $34(56 \%)$ & $47(76 \%)$ & $48(76 \%)$ & $57(90 \%)$ & $57(90 \%)$ \\
\hline Pictograms in first row & $24(39 \%)$ & $36(58 \%)$ & $40(63 \%)$ & $40(63 \%)$ & $40(63 \%)$ \\
\hline Pictograms in first position & $3(5 \%)$ & $2(3 \%)$ & $0(0 \%)$ & - & - \\
\hline Pictogram colour first & $43(70 \%)$ & $49(79 \%)$ & $48(76 \%)$ & $0(0 \%)$ & $0(0 \%)$ \\
\hline Pictogram among first 5 of its colour & $19(31 \%)$ & $38(61 \%)$ & $38(60 \%)$ & $38(60 \%)$ & $38(60 \%)$ \\
\hline First pictogram of its colour & $6(10 \%)$ & $12(19 \%)$ & $13(21 \%)$ & $13(21 \%)$ & $13(21 \%)$ \\
\hline Time needed & $19 \mathrm{~min}$. & $9 \mathrm{~min}$. & $8 \mathrm{~min}$. & $6 \mathrm{~min}$. & $6 \mathrm{~min}$. \\
\hline
\end{tabular}

pictograms that have been used when the pictograms of their colour were placed on top of the rest of the colours, meaning that the colour based prediction guesses correctly the colour of the pictogram that comes next.

Since not all the pictograms of the same POS colour fit into one screen, the rows "Pict among first 5 of its colour" and "First pictogram of its colour" show how many selected pictograms are placed among the first ones with the same colour, which makes it easier to find them. The proportion for both rows doubles after the first week and then remains stable, showing the same influence of the frequency based prediction over the colour based prediction that we could observe with the pictograms in the first screen and in the first row.

The last row shows the time needed by the evaluator to perform the tasks assigned for each week, reinforcing the intuition that we got with the previous results showing that the required time decreases when the system is already trained and the POS colour based prediction is turned off.

This suggests that a different kind of prediction is to be used in conjunction with the POS colour based prediction if we intend to take advantage of the colour of a pictogram to predict the next one to be used, since, as we can see in the table, the percentage of pictograms found in the first position of the screen is really low, as well as in the first position of its colour.

\subsubsection{Qualitative Results}

In addition to the results obtained from the analysis of the interaction of the evaluator with the application, a handful of valuable insights have also been acquired during the evaluation itself and while analysing the results.

The first interesting result is that, in contrast to what we expected, the POS colour of the pictograms was not especially relevant for the evaluator. The design of the ARASAAC pictograms made us expect that considering the POS colour categories they represent would be of use in the editor, but as the evaluator showed us, the fact that her students have a low cognitive level and their lack of ability to communicate with other people makes this feature a secondary one. In addition, the way of constructing the messages, using concepts more than sentences, made the categories other than nouns be of scarce relevance, which, in turn, caused the POS colour based prediction to be of little use in this context. This does not discard this feature as one to be taken into account, but its use must be further tested in other contexts where messages using pictograms are effectively built using sentences instead of a succession of concepts or events.

In terms of the interaction with the editor, there are two more facts that have drawn our attention with respect the way in which the prediction works. The first of them is the fact that the evaluator expected to find the pictograms in the same place she found them when she used them for the first time. Therefore, she was initially puzzled when the pictograms were in a different screen and so she had to look for them in a different place, taking longer to finish the searched pictograms in certain cases. This effect dimmed when the prediction algorithms placed the pictograms in the first screen, so they could be found at first glance, but otherwise the users are a bit lost until the behaviour of the editor stabilizes. The other one has to do with the implicit or- 
der that some pictograms may have among them. This could be seen when the evaluator had to write the date at the beginning of each day. In this case, the days of the week, of the month, the months and the years already have a numerical order, so changing this order to prioritize the frequency of use made the pictograms be unsorted, thus making it a little more difficult to find them, even if they were in the first screen. Consequently, in these cases it may be more suitable to try to keep the implicit order of the pictograms, at least within each screen of the editor.

Additionally, when using the frequency based prediction, the most commonly used pictograms tend to be moved to the first places of the list of pictograms. However, when a pictogram is used, the probability of using it again in the same message is almost 0 , so moving it to the first positions may not be the most suitable decision. Since the most frequently used pictograms usually fitted in the first screen, the effect of this decision was not noticeable, but a combination with an n-grams based prediction may provide a more effective suggestion for the next pictogram to use.

\section{Conclusions and Future Work}

In this paper we have presented PictoEditor, an Augmentative and Alternative Communication application for composing messages in pictograms, aimed at facilitating social communication directed at users on the autism spectrum. The main novelty of this tool is the use of prediction techniques in order to lessen the burden of finding the desired pictograms among a high number of choices. In order to assess the support offered by the tool, we have evaluated three different functionalities for facilitating composition of pictogram messages. The results show that prediction based on sequencing of syntactic categories of pictogram is not as important as expected, but the combination of prediction based on pictogram frequency and POS colour shortcuts are enough to present the most likely pictograms to the user after some usage.

From the point of view of its final users, PictoEditor is able to adapt to each person as the prediction statistics are learnt while the tool is used. This is very practical from the point of view of the final users. Whereas most applications are configured with general profiles of users that try to cover all possibilities for a specific impairment, in fact capabilities and individual preferences will surely not be the same for two different people even if the share the same disability.

The evaluation has been performed with a teacher of children with autism spectrum disorder. From the point of view of the prediction, the results were very good, with the evaluator requiring a significantly lower time (up to three times less) to write the pictogram messages when the system had learned during the process. This result is in line with other works on word prediction.

Due to the low cognitive level and communication problems of her students, it has not been possible to test the tool directly with the children. However, we have contacted some associations, like Confederación Autismo España, which work with a great number of users on the autism spectrum, in order to validate the application with end users. This kind of evaluation is quite complex, so we plan to rely on validated user experience questionnaires. However, when working with users with disabilities, it is important to be able to adjust these questionnaires to the difficulties of the user, so we will explore flexible solutions like the UEQ + framework (Schrepp and Thomaschewski 2019) and short questionnaires like UEQ-S (Schrepp et al. 2017).

Some lines of future work are open to improve the tool. From the point of view of the frequency and POS colour based prediction mechanisms, we could explore the use of more complex n-grams like bigrams and trigrams. Especially in the case of POS colour prediction, where the results obtained in the evaluation have shown that sometimes it is not very useful, we would test if the storage of more complex colour patterns (i.e. orangegreen-orange) could improve the prediction results. The application could also be improved by introducing context awareness, so depending on the place where the user is using the tool, or the task he is performing, the system could adapt to offer the most suitable pictograms (Alegre-Ibarra et al. 2018). Finally, an interesting addition could be the search of pictograms by name. This would obviously be the quickest way of finding a pictogram, but we must take into account that most users of this kind of tools are not able to use conventional language, so such a functionality would be more oriented to teachers or family who rely on the tool when working with final users.

Acknowledgements The work presented in this paper has been partially funded by the projects IDiLyCo: Digital Inclusion, Language and Communication, Grant. No. TIN201566655-R (MINECO/FEDER) and InVITAR-IA: Infraestructuras para la Visibilización, Integración y Transferencia de Aplicaciones y Resultados de Inteligencia Artificial, UCM Grant. No. FEI-EU-17-23. The pictograms used in this work are a property of Gobierno de Aragón and have been created by Sergio Palao for ARASAAC (http://arasaac.org). The pictograms are distributed under the Creative Commons license (BY-NC-SA). 


\section{References}

Alegre-Ibarra U, Augusto JC, Evans C (2018) Perspectives on engineering more usable context-aware systems. Journal of Ambient Intelligence and Humanized Computing 9(5):1593-1609

Allen M, Leung R, McGrenere J, Purves B (2008) Involving domain experts in assistive technology research. Universal Access in the Information Society $7(3): 145-154$

Allsop M (2010) Involving children in the design of healthcare technology. PhD thesis, U. of Leeds

Alonso-Virgos L, Pascual Espada J, Rodríguez Baena L, Crespo RG (2018) Design specific user interfaces for people with down syndrome using suitable wcag 2.0 guidelines. Journal of Ambient Intelligence and Humanized Computing 9(5):1359-1374

Ameen S, Vadera S (2017) A convolutional neural network to classify American Sign Language fingerspelling from depth and colour images. Expert Systems 34, DOI 10.1111/exsy.12197

Anson D, Moist P, Przywara M, Wells H, Saylor H, Maxime H (2006) The effects of word completion and word prediction on typing rates using on-screen keyboards. Assistive Technology 18(2):146-154

Arnott JL, Newell AF, Alm N (1992) Prediction and conversational momentum in an augmentative communication system. Communications of the ACM 35(5):46-57

Baldassarri S, Peña P, Cerezo E, Marco J (2014a) Estado del arte en sistemas de comunicación alternativa y aumentativa. AUTI: Aplicaciones y Usabilidad de Televisión Digital Interactiva.

Baldassarri S, Rubio JM, Azpiroz MG, Cerezo E (2014b) Araboard: A multiplatform alternative and augmentative communication tool. Procedia Computer Science 27:197-206

Bautista S, Hervás R, Hernández-Gil A, Martínez-Díaz C, Pascua S, Gervás P (2017) Aratraductor: Text to pictogram translation using natural language processing techniques. In: Proc. of the 18th Intl. Conf. of the Spanish HCI Association (Interacción 2017)

Frauenberger C, Good J, Keay-Bright W (2011) Designing technology for children with special needs: bridging perspectives through participatory design. CoDesign 7(1):1-28

Garay-Vitoria N, Abascal J (2006) Text prediction systems: a survey. Universal Access in the Information Society 4(3):188-203

Garay-Vitoria N, Abascal J, Urigoitia-Bengoa S (1995) Application of the human conversation modelling in a telephonic aid. In: Proceedings of the 15th International Symposium on Human Factors in Telecommu- nications (HFT'95), pp 131-138

Garcia L, de Oliveira L, de Matos D (2014) Word and sentence prediction: Using the best of the two worlds to assist aac users. Technology and Disability 26(2, 3):79-91

Garcia LF, Oliveira LCD, Matos DMD (2015) Measuring the performance of a location-aware text prediction system. ACM Trans Access Comput 7(1):2:12:29, DOI 10.1145/2739998

Garcia LF, de Oliveira LC, de Matos DM (2016) Evaluating pictogram prediction in a location-aware augmentative and alternative communication system. Assistive Technology 28(2):83-92

García P, Lleida E, Castán D, Marcos JM, Romero D (2015) Context-aware communicator for all. In: International Conference on Universal Access in HumanComputer Interaction, pp 426-437

Gea M, Rodríguez M, Rodríguez M, Medina N, LópezCózar R, Paderewski P, Gutiérrez F (2006) Adaptive and context-aware hypermedia model for users with communication disabilities. In: Navarro-Prieto R, Vidal JL (eds) HCI related papers of Interacción 2004, Springer Netherlands, Dordrecht, pp 19-28

Hervás R, Francisco V, Méndez G, Bautista S (2019) A user-centred methodology for the development of computer-based assistive technologies for individuals with autism. In: Lamas D, Loizides F, Nacke L, Petrie H, Winckler M, Zaphiris P (eds) Human-Computer Interaction - INTERACT 2019, Springer International Publishing, Cham, pp 85-106

Higginbotham DJ, Bisantz AM, Sunm M, Adams K, Yik F (2009) The effect of context priming and task type on augmentative communication performance. Augmentative and Alternative Communication 25(1):19-31

Holone H, Herstad J (2013) Three tensions in participatory design for inclusion. In: Proceedings of the SIGCHI Conference on Human Factors in Computing Systems, ACM, CHI '13, pp 2903-2906

Keay-Bright W (2007) The reactive colours project: demonstrating participatory and collaborative design methods for the creation of software for autistic children. Design - Principles \& Practices 1(2):7-15

Khari M, Garg AK, Crespo RG, Verdú E (2019) Gesture Recognition of RGB and RGB-D Static Images Using Convolutional Neural Networks. International Journal of Interactive Multimedia and Artificial Intelligence 5(7):22-27

Kientz JA, Hayes GR, Westeyn TL, Starner T, Abowd GD (2007) Pervasive computing and autism: Assisting caregivers of children with special needs. IEEE Pervasive Computing 6(1):28-35 
Kim G, Park J, Han M, Park S, Ha S (2009) Contextaware communication support system with pictographic cards. In: Proceedings of the 11th International Conference on Human-Computer Interaction with Mobile Devices and Services, ACM, New York, NY, USA, MobileHCI '09, pp 86:1-86:2

Koester HH, Levine S (1996) Effect of a word prediction feature on user performance. Augmentative and Alternative Communication 12(3):155-168

Magnuson T, Hunnicutt S (2002) Measuring the effectiveness of word prediction: The advantage of long-term use. Speech, Music and Hearing Quarterly Progress and Status Report 3(1):57-67

Martín A, Hervás R, Méndez G, Bautista S (2018) Pictar: Una herramienta de elaboracin de contenido para personas con tea basada en la traducción de texto a pictogramas. In: Proceedings of the 19th International Conference on Human-Computer Interaction (Interacción 2018)

Mitchell M, Sproat R (2012) Discourse-based modeling for aac. In: Proc. of the Third Workshop on Speech and Language Processing for Assistive Technologies, Association for Computational Linguistics, pp 9-18

Pahisa Sol J (2017) From telegraphic to natural language: an expansion system in a pictogram based aac application. PhD thesis, Departament d'Enginyeria de la Informaci i de les Comunicacions

Pantsar-Syväniemi S, Ervasti M, Karppinen K, Väätänen A, Oksman V, Kuure E (2015) A situationaware safety service for children via participatory design. Journal of Ambient Intelligence and Humanized Computing 6(2):279-293

Pouplin S, Robertson J, Antoine JY, Blanchet A, Kahloun JL, Volle P, Bensmail D (2014) Effect of a dynamic keyboard and word prediction systems on text input speed in patients with functional tetraplegia. Journal of rehabilitation research and development 51(3):467-480

van Rijn H, Stappers PJ (2008) Expressions of ownership: motivating users in a co-design process. In: Proc. of the Tenth Anniversary Conf. on Participatory Design 2008, Indiana University, pp 178-181

San-Segundo R, Montero JM, Córdoba R, Sama V, Fernández F, D'Haro LF, López-Ludeña V, Sánchez D, García A (2012) Design, development and field evaluation of a Spanish into sign language translation system. Pattern Analysis and Applications 15(2):203-224

Schrepp M, Thomaschewski J (2019) Design and Validation of a Framework for the Creation of User Experience Questionnaires. International Journal of Interactive Multimedia and Artificial Intelligence 5(7):8895
Schrepp M, Hinderks A, Thomaschewski J (2017) Design and Evaluation of a Short Version of the User Experience Questionnaire (UEQ-S). International Journal of Interactive Multimedia and Artificial Intelligence 4(6):103-108

Sevilla AF, Lahoz-Bengoechea JM (2019) A different description of orientation in sign languages. Procesamiento del Lenguaje Natural 62:53-60

Tao W, Leu MC, Yin Z (2018) American Sign Language alphabet recognition using Convolutional Neural Networks with multiview augmentation and inference fusion. Engineering Applications of Artificial Intelligence 76:202 - 213

Trnka K, Yarrington D, McCoy K, Pennington C (2006) Topic modeling in fringe word prediction for aac. In: Proceedings of the 11th International Conference on Intelligent User Interfaces, ACM, New York, NY, USA, IUI '06, pp 276-278

Trnka K, Yarrington D, McCaw J, McCoy KF, Pennington C (2007) The effects of word prediction on communication rate for aac. In: Human Language Technologies 2007: The Conference of the North American Chapter of the Association for Computational Linguistics; Companion Volume, Short Papers, Association for Computational Linguistics, Stroudsburg, PA, USA, NAACL-Short '07, pp 173-176

Venkatagiri H (1993) Efficiency of lexical prediction as a communication acceleration technique. Augmentative and Alternative Communication 9(3):161-167

Verdú E, Bustelo CPG, Martínez MA, Crespo RG (2017) A System to Generate SignWriting for Video Tracks Enhancing Accessibility of Deaf People. International Journal of Interactive Multimedia and Artificial Intelligence 4(6):109-115

Wiegand K, Patel R (2012) Non-syntactic word prediction for aac. In: Proceedings of the Third Workshop on Speech and Language Processing for Assistive Technologies, Association for Computational Linguistics, pp 28-36 